\title{
Ocular vergence-induced accommodation and its relation to dark focus
}

\author{
R. J. MILLER \\ Washington State University, Pullman, Washington 99164
}

\begin{abstract}
Two experiments were conducted in an attempt to develop an open-loop technique for assessing the influence of ocular vergence on accommodation changes. Past research had shown that a small point of light $(1.2 \mathrm{~mm}$ in diameter) induces relatively small changes in monocular accommodation at viewing distances greater than $50 \mathrm{~cm}$. However, Experiment 1 (using seven male and six female undergraduates as subjects) demonstrated that, even with a smaller light spot $(.29 \mathrm{~mm})$, subjects could accommodate to the stimulus at distances closer than $50 \mathrm{~cm}$. It was only with a very impoverished target $(.08 \mathrm{~mm}$ diameter), as used in Experiment 2 (in which five male and five female undergraduates served as subjects), that it was possible to present a stimulus that could induce ocular vergence without being a direct stimulus in itself for accommodation. It was also shown that ocular vergence can drive accommodation and that ocular vergence can vary systematically under conditions in which illumination is greatly reduced. In addition, both experiments showed significant relationships among dark focus and both monocular and binocular accommodation.
\end{abstract}

Several recent studies (e.g., Malmstrom \& Randle, 1976; Provine \& Enoch, 1975) have addressed the issue of the degree to which accommodation can be voluntarily controlled. However, the effects of ocular vergence typically have not been examined in such studies, making conclusions regarding the voluntary nature of accommodation difficult to evaluate. If, as several writers (e.g., Fincham \& Walton, 1957; see Morgan, 1968, for a review) have suggested, changes in ocular vergence automatically produce changes in accommodation, it is possible that the voluntary response is actually ocular vergence (which is known to have voluntary components) and that accommodation changes just follow along. If such were the case, it would hardly be correct to describe accommodation as directly under voluntary control.

It was the above problem that initially stimulated the present study. To investigate the relationship between ocular vergence and accommodation, a technique was required in which vergence could be controlled by the experimenter, with accommodation free to vary over its entire range (i.e., what Morgan, 1968 , referred to as an open-loop condition). That is, a stimulus that would reliably induce vergence changes but not serve as a stimulus in itself for accommodation was required. It could thus be inferred that any changes in accommodation could result from changes in ocular vergence rather than from sensory information provided by the stimulus.

The author expresses his appreciation to Concetta FoschiniMiller, Robert LeBeau, Helen Heppel, and Kenneth Gish for their assistance in conducting these experiments, and to Herschel W. Leibowitz and D. Alfred Owens for reviewing the manuscript. Reprint requests should be addressed to R. J. Miller, Department of Psychology, 350 Johnson Tower, Washington State University, Pullman, Washington 99164.
The need for such an open-loop procedure has long been recognized. Fincham and Walton (1957) described the "need to eliminate the direct stimulation of accommodation ... while at the same time presenting to each eye sufficiently well-defined images to stimulate binocular fusion and to control convergence" (p. 489). Indeed, Fincham and Walton developed a procedure for doing so. They used a modified coincidence optometer for measuring accommodation and had their subjects converge on a $.5-\mathrm{mm}$ light spot. However, such an apparatus is costly, requires careful adjustments for each subject's interpupillary distance, and presents the risk of inducing instrument myopia (see Hennessy, 1975). In addition, as will be shown, the assumption that a .5 -mm light spot does not induce accommodation may not have been valid.

A relatively inexpensive and simple technique for inducing convergence without providing a direct sensory cue to accommodation appeared to be provided by recent research on the dark focus of accommodation. Dark focus (DF) refers to the refractive power of the eye in the absence of an external stimulus for accommodation, as is the case, for example, in total darkness. Research, much of it conducted using the laser optometer, has shown that as illumination is decreased, accommodation shifts from a focus that is more or less appropriate to the stimulus distance to an intermediate DF (for reviews, see Leibowitz \& Hennessy, 1975; Leibowitz \& Owens, 1975, 1978; Miller, 1978a, 1978b). Accommodation also shifts toward DF when the observer looks through a small artificial pupil, thereby increasing depth of field of the eye and making accommodation unnecessary (Hennessy, lida, Shiina, \& Leibowitz, 1976; Leibowitz, Hennessy, \& Owens, 1975; Leibowitz 
\& Owens, 1975), as well as when the observer is looking into a Ganzfeld (Leibowitz \& Owens, 1975; Westheimer, 1957; Whiteside, 1952). More important for the present study, Owens and Leibowitz (1975) have shown that a pinpoint light source of $1.2-\mathrm{mm}$ diam does not present a very effective stimulus to induce accommodation. Rather, when viewing such a target monocularly in an otherwise totally dark room, their subjects' accommodation tended to revert to the DF position regardless of whether the target was presented at a distance of 50 or $400 \mathrm{~cm} .^{1}$

Because of the findings of Fincham and Walton (1957) and Owens and Leibowitz (1975) using a small target, it appeared to the present author that such a stimulus would be ideal for controlling convergence without directly affecting accommodation. The idea was to present the target in otherwise total darkness at various distances binocularly, require subjects to converge on the target, and measure accommodation. However, it was discovered early in the process of developing such a procedure that it had a major problem. Owens and Leibowitz (1975) did not measure accommodation to stimuli any closer to the observer than $50 \mathrm{~cm}$. But pilot work by the present author showed very quickly that at distances closer than $50 \mathrm{~cm}$ some observers, viewing monocularly, could definitely accommodate to such a stimulus. One of the principal purposes of the present study, then, was to develop a target that would be an effective ocular vergence stimulus while at the same time not provide adequate stimulation for accommodation at any distance from the observer.

Another purpose of the present study was to examine to some degree the general relationships among ocular vergence, DF, and general accommodation. These relationships, particularly with regard to DF, have received relatively little attention. Owens and Leibowitz (1976) examined DF and ocular vergence in relation to the specific distance tendency (Gogel, 1972) and found that perceived distance is related to the resting position of convergence. However, they did not examine in much detail the more general relationship of ocular vergence to DF. One important question concerns the tendency of changes in ocular vergence to influence accommodation. It is assumed by some researches that convergence can drive accommodation (Fincham \& Walton, 1957; Morgan, 1968). In fact, Fincham and Walton have maintained that convergence is the primary stimulus to dynamic accommodation in normal binocular vision. However, the relationship between the two variables is by no means clear, and there appear to be relatively wide individual differences in the degree to which ocular vergence drives accommodation, with the widest differences observed at close convergence distances (see Morgan, 1968, for a review). Another important question involves the effects of reduced illumination on ocular vergence. Fincham (1962), Ivanoff (1955), and
Owens and Leibowitz (1976) have reported that, under reduced illumination, ocular vergence assumes an intermediate resting position, analogous to DF (although apparently not identical to it-see Fincham, 1962, and Owens \& Leibowitz, 1976). The present study presented an opportunity to examine this effect as well.

\section{EXPERIMENT 1}

\section{Method}

\section{Apparatus}

Basic visual functioning was assessed for all subjects using a Bausch and Lomb master Orthorater. The subjects were required to have far acuity equal to or better than 20/20 and near acuity equal to or better than $13 / 13$. In addition, no subject was used who showed any phoria outside of the following ranges: far vertical, $\pm .5^{\Delta}$; far lateral, $\pm 3.0^{\Delta}$; near vertical, $\pm .5^{\Delta}$; near lateral, $-7.0^{\Delta}$ to $+4.0^{\Delta}$. Subjects who normally wore corrective lenses wore them during the Orthorater measurements and in all phases of the study.

Optometer. Accommodation was measured with a laser optometer, various stages and refinements in the development and use of which have been reported by several researchers (Hennessy \& Leibowitz, 1970, 1972; Leibowitz \& Hennessy, 1975; Leibowitz \& Owens, 1978). The optometer used in the present experiment was identical to that described by Miller (1978b), as diagrammed in Figure 1. A beam of light from a low-output laser $(2.5 \mathrm{~mW} \mathrm{He}-\mathrm{Ne}$, Metrologic ML920) was diverged with a +20-D lens and reflected from the surface of a slowly revolving drum $(.63 \mathrm{rpm}$, diameter = $6.60 \mathrm{~cm}$ ). The reflected pattern was then superimposed in the subject's left monocular visual field for durations of $.5 \mathrm{sec}$. The pattern was seen by the subject as a moving speckle pattern, with perceived movement determined by the movement of the drum and the subject's accommodative state. When the subject was overaccommodated for the optical distance of the drum, the speckles were seen as moving in the same direction as the drum's rotation. The opposite was true when the subject was underaccommodated. When the subject was accommodated at the optical distance of the drum, the speckles appeared to move in slow random swirls or not to move at all. Hennessy and Leibowitz (1970) showed that .5-sec exposures of such a stimulus do not affect accommodation.

One focal length $(20 \mathrm{~cm})$ from the subject's eye, a +5-D lens was inserted into the path of the speckle pattern. The drum could be slid along an optical bench and its optical distance thus varied from infinity $(0 \mathrm{D})$ to $20 \mathrm{~cm}(+5 \mathrm{D})$ without producing any change in the apparent brightness or size of the speckle pattern (the Badal principle; see Hennessy \& Leibowitz, 1972; Ogle, 1968).

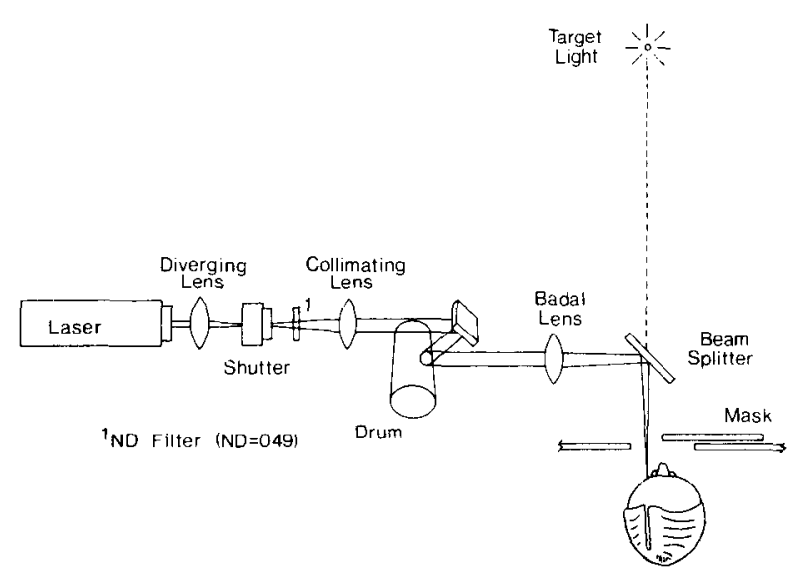

Figure 1. Diagram of laser optometer, indicating location of mask (shown half open) and target light. 
However, the actual distance of the drum from the subject varied over a range of only $20 \mathrm{~cm}$.

Target light. Light for the target was provided by a dc bulb (GE 222, powered at $2.5 \mathrm{~W}$ ) with a luminous intensity of about $10.0 \mathrm{~cd}$. A pinhole (Diameter $=.29 \mathrm{~mm}$ ) was mounted $13 \mathrm{~mm}$ in front of the bulb. The visual angle subtended by the target varied from $.0042 \mathrm{deg}(.000073 \mathrm{rad})$ to $.067 \mathrm{deg}(.0012 \mathrm{rad})$, depending on its distance from the subject. The target light was mounted in a small box that could easily be moved to the various distances required. The distances chosen for examination were $25,33,50$, 100 , and $400 \mathrm{~cm}$. These viewing distances were chosen because they correspond to $4.0,3.0,2.0,1.0$, and $.25 \mathrm{D}$ of accommodation, respectively. Regardless of distance, the target light was always located directly in line with the subject's left eye (see Figure 1) so that when the laser pattern was flashed, it always was superimposed on the pinpoint light spot. Neutral density filters were placed in front of the target light to ensure that the luminous flux of the light remained the same at all distances.

Mask. In front of the subject's eyes (see Figure 1) was mounted a wooden panel that could be slid back and forth in front of the subject. When this mask was open, the subject could see the target light with both eyes. When the mask was half-open, the subject's right-eye view of the target light was blocked so that he could see it only with his left eye. This mask could also be closed entirely so that the subject could see nothing of the target light.

\section{Subjects}

The subjects were 13 students ( 7 males and 6 females) enrolled in introductory psychology courses at Washington State University; they were volunteers who received extra points toward their psychology course grades as compensation for their participation. They ranged in age from 18 through 23 years $(M=19.9$ years).

\section{Procedure}

Practice session. Experience with the laser optometer has shown that most subjects require some practice to see the speckle pattern reliably. During the practice session, the subject was shown the laboratory and the optometer, was given careful instructions regarding what to look for when the speckle pattern was flashed, and then was given practice in looking at the speckle pattern and determining its direction of movement. When it became clear that the subject was perceiving the movement of the speckle pattern reliably, he/she was scheduled for the next session and dismissed. At the end of the practice session, each subject was cautioned not to use drugs, medications, alcohol, etc., on the day of the next session.

Experimental session. When the subject arrived for this session, he/she was seated at the optometer, and a head-and chinrest was adjusted to place the speckle pattern in the subject's line of vision. The mask remained open. All lights were then extinguished, and a few practice trials were conducted to ensure that the subject could still detect reliably the movement of the speckle pattern. A "bracketing" technique was then used to find the DF point. The speckle pattern was flashed for $.50 \mathrm{sec}$ at irregular intervals, preceded each time by a signal of "ready" from the experimenter. After each flash, the subject indicated whether he/she perceived the movement of the speckles to be upward or downward. Following each response, the drum was moved to a different position on the optical bench. At the beginning of the series of flash presentations, the drum position alternated randomly between extreme far and near optical distances (thus "bracketing" the DF point). With succeeding presentation, the distance between near and far flashes was gradually reduced, $1 \mathrm{~cm}$ at a time, until the point of no apparent motion was found.

This value, expressed in diopters, was recorded at the DF point, although two correction factors were required. "Since laser light is monochromatic, a small constant compensating for axial chromatic aberration of the eye $(+.33 \mathrm{D}$, assuming $560 \mathrm{~nm}$ as the reference wavelength) must be added to each measure to obtain the absolute accommodation" (Owens \& Leibowitz, 1975). In addition, the optometer was so constructed that the optical distance of the DF point was measured to the surface of the drum. Charman (1974) has shown that when a curved surface is used. the effective plane of stationarity is actually between the surface of the curve and its axis of rotation. Applying Charman's formula, this plane was calculated to be $1.99 \mathrm{~cm}$ from the drum's surface, and this correction was included in the final conversion.

Following the determination of DF, the mask was closed and a table lamp was turned on. Then the target light (with appropriate neutral density filters) was placed at one of the five distances indicated earlier, specific distance being determined randomly. Since the mask was closed, the subject could not see where the light was being placed. In addition, an attempt was made by the experimenter to make the same movement for each distance, so that the subject could not determine the location of the light by sound cues. This was felt to be an important control, since research has shown that both accommodation and ocular vergence can be influenced by the known distance of the target (Morgan, 1968). Then the room was again made dark, and the mask was opened either halfway (if it was to be monocular condition) or all the way (if it was to be a binocular condition). The subject was then instructed to look at the white spot of light and to "keep it in clear focus at all times." If it was a binocular condition, the subject was reminded to "be especially careful to keep it as a single image. Do not let your eyes wander so that you see a double image of the spot." As the subject looked at the target light, his/her accommodation was assessed with the optometer using the same procedure used previously to find the DF point.

The above procedure was repeated for all 10 ocularity-viewing distance combinations, the order of presentation of the 10 conditions being determined randomly. Thus, for each subject, a total of 11 accommodation values were recorded, one for the initial DF baseline measure and one for each of the 10 ocularity-viewing distance conditions. Following completion of all measurements, the subject was given a brief explanation of the goals and purposes of the experiment and dismissed.

\section{Results}

Mean accommodation values for all subjects in each of the 10 ocularity-viewing distance combinations are graphed in Figure 2. The mean DF value for all 13 subjects was $1.20 \mathrm{D}(\mathrm{SD}=.63)$.

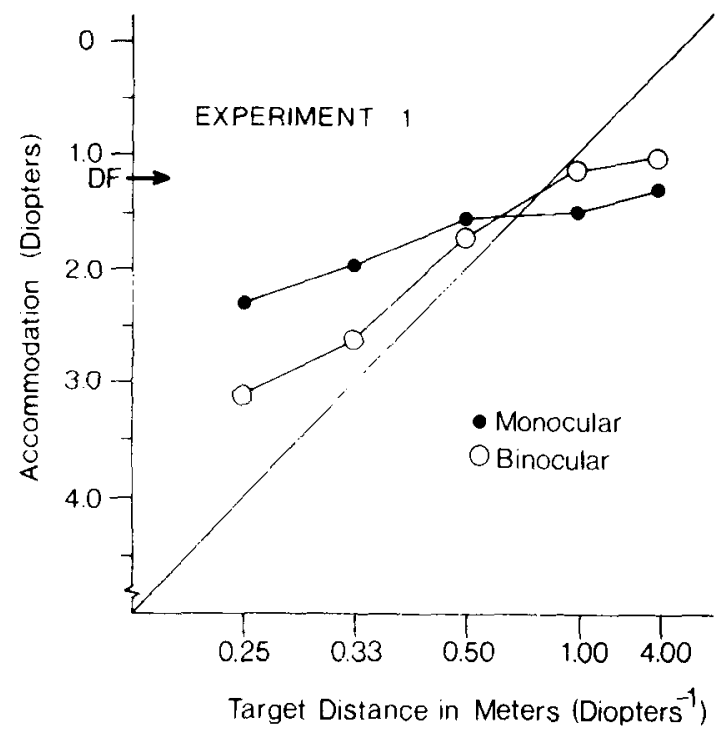

Figure 2. Summary of accommodation results from Experiment 1 for monocular and binocular viewing at all five target-light distances. Arrow indicates mean DF for all subjects (1.2 D). 


\section{Influence of Target Distance on Monocular} and Binocular Accommodation

As stated in the introduction, one of the principal purposes of the present experiment was to create a stimulus condition that would induce changes in ocular vergence without directly affecting accommodation. However, the results of Experiment 1 showed that both binocular and monocular accommodation were influenced by target distance.

A 2 by 5 (Ocularity by Target Distance) analysis of variance was performed, with the dependent variable being each subject's accommodation under each of the 10 viewing conditions (see Figure 2). The overall effect due to ocularity was not statistically significant. There was a significant overall effect of target distance on accommodation $[F(4,48)=43.07$, $\mathrm{p}<.01$ ]. That is, the closer target distances produced greater accommodation than did the farther target distances.

There was also a significant interaction between ocularity and target distance $[F(4,48)=6.89, p<.01]$. Because of this interaction, a simple main effects analysis was performed. This analysis showed that the effects of target distance on accommodation were significant both for monocular viewing $[F(4,48)=$ $7.68, \mathrm{p}<.01]$ and for binocular viewing $[\mathrm{F}(4,48)=$ $44.04, p<.01]$. Thus, it is clear that although target distance had a greater effect on accommodation when there were vergence cues present (binocular viewing) than when the target was viewed monocularly, nevertheless target distance had a significant impact on accommodation under both conditions.

A more direct demonstration of the differential effects of monocular and binocular viewing on accommodation can be seen in another, somewhat different analysis. The diagonal line in Figure 2 represents a perfect one-to-one relationship between accommodation and viewing distance, assuming a subject was accurately accommodated for each of the five target distances. It is readily apparent from examination of Figure 2 that the curve corresponding to binocular viewing is more nearly parallel to this diagonal than is the curve corresponding to monocular viewing. A 2 by 5 (Ocularity by Viewing Distance) analysis of variance was performed, with the dependent variable being the absolute difference (i.e., ignoring signs) between each subject's actual accommodation and the target distance (in accommodation diopters) under each of the 10 viewing conditions. Results of this analysis showed a significant effect due to ocularity $[F(1,12)=14.78$, $\mathrm{p}<.01]$. In other words, target distance exerted a significantly stronger overall influence on binocular than on monocular accommodation. The effect of target distance was also significant $[\mathrm{F}(4,48)=5.01$, $\mathrm{p}<.01]$, as was the interaction $[\mathrm{F}(4,48)=2.82$, $\mathrm{p}<.05]$.

Based on the results of the first analysis of variance
Table 1

Results of Newman-Keuls Analyses for Experiment 1

\begin{tabular}{|c|c|c|c|c|}
\hline \multirow[b]{2}{*}{ Distance } & \multicolumn{4}{|c|}{ Viewing Distance } \\
\hline & 100 & 50 & 33 & 25 \\
\hline & \multicolumn{4}{|c|}{ Tests for Monocular Viewing } \\
\hline $\begin{array}{r}400 \\
100 \\
50 \\
33\end{array}$ & .18 & $\begin{array}{l}.26 \\
.08\end{array}$ & $\begin{array}{l}.62^{*} \\
.44 \\
.36\end{array}$ & $\begin{array}{l}.96^{*} \\
.78^{* *} \\
.70^{*} \\
.34\end{array}$ \\
\hline 33 & \multicolumn{4}{|c|}{ Tests for Binocular Viewing } \\
\hline $\begin{array}{r}400 \\
100 \\
50 \\
33\end{array}$ & .11 & $\begin{array}{l}.69^{* *} \\
.59^{*}\end{array}$ & $\begin{array}{r}1.61^{* *} \\
1.50^{* *} \\
.91^{* *}\end{array}$ & $\begin{array}{c}2.09 * * \\
1.98 * * \\
1.39 * * \\
.48\end{array}$ \\
\hline
\end{tabular}

Note-Distances are given in centimeters. Tabled values represent differences between pairs of means. Standard error $=.14$ in all cases. $\quad{ }^{*} p<.05 . \quad{ }^{* *} p<.01$.

(using each subject's accommodation under each of the 10 viewing conditions as the dependent variable), Newman-Keuls a posteriori tests were performed for both the monocular and binocular conditions. The results of these analyses are shown in Table 1 . As can be seen, the effects of target distance on accommodation are quite consistent over most of the distance range for binocular viewing. On the other hand, the principle differential effects of target distance on monocular accommodation appear to be limited to the near viewing distances; the difference between 25 and $50 \mathrm{~cm}$ is significant, while that between 50 and $400 \mathrm{~cm}$ is not.

\section{Relationships Between DF and Monocular and Binocular Accommodation}

As stated in the introduction, another purpose of the present study was to examine the relationships among DF and monocular and binocular accommodation to the target. Accordingly, for each of the 10 ocularity-viewing distance conditions, correlations were examined using subjects' DF baseline values as one variable and their actual accommodation scores as the other. The resulting 10 Pearson productmoment correlation coefficients are shown on the left side of Table 2, under Experiment 1. As can be seen, monocular accommodation was also significantly related to DF at all viewing distances. At far viewing distances (i.e., 100 and $400 \mathrm{~cm}$ ), binocular accommodation was also significantly related to DF. It is clear that DF was related to both monocular and binocular accommodation. It is also clear that the relationship to DF was stronger for monocular than for binocular accommodation. This can be seen by examining the correlation coefficients in Table 1, but it was also confirmed in another manner. A 2 by 5 (Ocularity by Target Distance) analysis of variance was performed, with the dependent variable being the absolute difference (i.e., signs ignored) between each subject's accommodation at a given condition and that sub- 
Table 2

Pearson Product-Moment Correlations Between DF Baseline and Accommodation at Each Target Distance

\begin{tabular}{ccccc} 
& \multicolumn{4}{c}{ Viewing } \\
\cline { 2 - 5 } Target & \multicolumn{2}{c}{ Experiment 1} & \multicolumn{2}{c}{ Experiment 2} \\
\cline { 4 - 6 } Distance & Monocular & Binocular & Monocular Binocular \\
\hline $25(4 \mathrm{D})$ & $.754^{* *}$ & -.163 & $.897^{* *}$ & .450 \\
$33(3 \mathrm{D})$ & $.730^{* *}$ & .473 & $.913^{* *}$ & $.859^{* *}$ \\
$50(2 \mathrm{D})$ & $.546^{*}$ & .448 & $.740^{* *}$ & $.615^{*}$ \\
$100(1 \mathrm{D})$ & $.505^{*}$ & $.643^{* *}$ & $.896^{* *}$ & $.934^{* *}$ \\
$400(.25 \mathrm{D})$ & $.833^{* *}$ & $.586^{*}$ & $.943^{* *}$ & $.719^{* *}$ \\
Mean & .674 & .397 & .878 & .715 \\
\hline
\end{tabular}

Note-All significance tests are one-tailed. All distances are given in centimeters. For Experiment $1, n=13$ and $d f=11$. For Experiment 2, $n=10$ and $d f=8 . \quad * p<.05 . \quad * p<.01$.

ject's baseline DF. The results of this analysis showed a significant effect due to ocularity $[F(1,12)=7.90$, $\mathrm{p}<.02$ ]. In other words, binocular viewing led to accommodation values significantly more divergent from DF than did monocular viewing. The analysis also showed a significant effect due to target distance $[F(4,48=21.71, p<.01]$ and a significant interaction $[\mathrm{F}(4,48)=4.11, \mathrm{p}<.01]$.

\section{Discussion}

As stated in the introduction, the principal goal of this study was to develop a technique that would provide an adequate stimulus for convergence without providing a direct stimulus for accommodation. It was clear that Experiment 1 was not successful in doing this. While it was true that the distance of the target exerted a greater effect on binocular than on monocular accommodation, monocular accommodation was clearly not independent of these distance effects. The subjects were able to accommodate monocularly to some degree to the target, especially at close viewing distances. At $50 \mathrm{~cm}$ and beyond, the results of Experiment 1 were consistent with those of Owens and Leibowitz (1975) - monocular accommodation was significantly correlated with DF. However, confirming the results of pilot work mentioned in the introduction, the target exerted a greater influence on monocular accommodation as it came closer than $50 \mathrm{~cm}$. This was true despite the fact that the target light was much smaller than that used by either Owens and Leibowitz (1975) or Fincham and Walton (1957). Because of these findings, it became obvious that the target parameters should be further explored, and this was the purpose of Experiment 2.

\section{EXPERIMENT 2}

Method
Apparatus
Most of the apparatus for Experiment 2 was the same as that
for Experiment 1. The subjects were screened for visual func-

tioning using the same procedure and same criteria, and the laser optometer was used. There were, however, two important apparatus changes introduced:

Target light. The target light for Experiment 2 used a pinhole of about $.08 \mathrm{~mm}$ diameter. The visual angle subtended by the target varied from $.0012 \mathrm{deg}(.000020 \mathrm{rad})$ to $.018 \mathrm{deg}(.00032 \mathrm{rad})$, depending on its distance from the subject. Since the pinhole was so small, a bright light source was needed to make the pinpoint of light visible at all viewing distances. The pinhole was mounted $14.5 \mathrm{~cm}$ in front of the filament of a Viewlex (Model AP-20) projector, which used a Radiant CBC bulb (75 W, about $750 \mathrm{~cd}$ of luminous intensity). The entire system was surrounded by a light-tight case, so that the only light that escaped came from the pinhole itself. In addition, a 1/8-in. $(.32 \mathrm{~cm})$ thick sheet of ground glass was placed $2.0 \mathrm{~mm}$ directly in front of the pinhole (i.e., on the observer's side) to make the spot of light even less resolvable. The result was a very tiny, very dim spot of light that had gradually decreased illuminance at its edges and essentially no resolvable boundaries. As was the case in Experiment 1, the target light was portable and could be moved to the various viewing distances easily.

White noise. In Experiment 1 , the attempt had been made to avoid giving subjects auditory cues regarding the location of the target light. This procedure took the form of attempting, when the light was being moved from position to position, to make more or less the same sounds for every condition. However, a few of the subjects in Experiment 1 indicated that this technique had been only partially successful and that they had occasionally been aware of whether the target light was going to be relatively close or far away because of the sounds made in moving it.

To ensure that no auditory information regarding location of the target light was available to subjects in Experiment 2, subjects wore a light, comfortable headset with foam rubber padding around the ears. During those periods when the position of the target light was being changed, white noise was presented through this headset at a volume that was not uncomfortably high $(78 \mathrm{~dB})$, but that was sufficient to mask the sounds of moving the light. This turned out to be a successful technique, as all subjects reported after the experiment that they had obtained no auditory information whatsoever regarding the location of the target light and that the volume of the noise was not uncomfortable.

\section{Subjects}

The subjects were 10 students ( 5 males and 5 females) recruited from introductory psychology courses at Washington State University. As in Experiment 1 , the subjects were volunteers who received extra points toward their psychology course grades as compensation for their participation. They ranged in age from 18 through 20 years $(M=19.1$ years). Initially, six male and six female subjects were recruited, but, for reasons that will be described later, one of each was excluded from data analyses.

\section{Procedure}

Practice session. Each subject reported to the laboratory for a practice session during which he/she learned how to report movement of the optometer speckle pattern. The procedure for this was the same as in Experiment 1.

Experimental session. The procedure for this session was very similar to that of Experiment 1. The target light was displayed to the subject under each of the same 10 ocularity-viewing distance combinations, presented in random order, each time with instructions to "keep the white spot of light in clear single-image focus at all times. It was especially emphasized to subjects that they should avoid allowing the target light to become a double image.

There were, however, two minor modifications in the procedure for Experiment 2. For one thing, whenever the target light was being moved, the white noise was turned on in the headset. During these times, the subject was instructed to sit back and close his/her eyes. Since the target light was very dim, it was felt that having subjects keep their eyes closed while the posi- 
tion of the target light was being changed (during which time a table lamp was on) would help somewhat in maintaining dark adaptation and make it easier for the subject to see the target light when it was exposed. Of course, as was the case in Experiment 1 , the room was totally dark when accommodation measurements were being made.

The other modification involved the manner in which baseline DF was measured. In Experiment 1, DF was measured at the beginning of the experimental session, followed by accommodation measurements for each of the 10 ocularity-target distance combinations. In Experiment 2, DF was measured twice, once at the beginning of the experimental session, as in Experiment 1, and once again after all 10 of the ocularity-target distance conditions had been presented. This was done because it was deemed desirable to determine if there were any fatigue effects reflected in DF.

\section{Results}

Of the initial 12 subjects who participated in Experiment 2, two were excluded. One male reported to the experimental session in a condition of high fatigue. Despite the fact that he had previously met all the visual criteria and had been able to detect the movement of the speckle pattern reliably during the practice session, he could not do so during the experimental session. When it became evident that he could not see the speckle pattern reliably, he was dismissed. One female subject had difficulty converging on the target light, although she had shown no sign of serious phorias during Orthorater screening. She appeared to be confused by the task, and the image of the target light seemed to alternate from one eye to the other during the binocular conditions. Her data were thus judged to be unreliable and so were not included in the analyses.

Mean accommodation values for the remaining 10 subjects across the 10 ocularity-viewing distance combinations are graphed in Figure 3. The difference

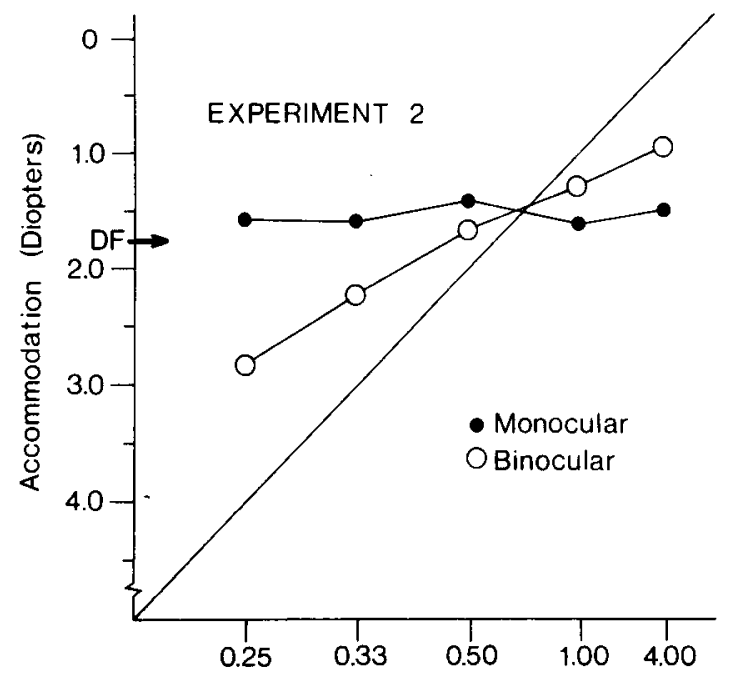

Target Distance in Meters (Diopters ${ }^{-1}$ )

Figure 3. Summary of accommodation results from Experiment 2 for monocular and binocular viewing at all five target-light distances. Arrow indicates mean DF for all subjects (1.76 D). between the two DF measures for the 10 subjects was not statistically significant $[t(9)=.76, p>.05]$. Indeed, in no case did the differences between the two measurements for any subject exceed $.5 \mathrm{D}$. As a result, each subject's DF was expressed as the mean of these two values. Using the resulting value, the overall mean $\mathrm{DF}$ for the 10 subjects was $1.76 \mathrm{D}(\mathrm{SD}=.95)$.

Unlike Experiment 1, the effects of target distance on binocular accommodation were quite different from the effects of distance on monocular accommodation (see Figure 3). A 2 by 5 (Ocularity by Viewing Distance) analysis of variance, with the dependent variable being each subject's accommodation under each of the 10 viewing conditions, revealed no significant effect for ocularity $[F(1,9)=3.68$, $\mathrm{p}>.05]$, a significant effect for target distance $[F(4,36)=26.07, p<.01]$, and a significant interaction $[F(4,36)=13.69, p<.01]$. Because of the interaction, a simple main effects analysis was performed. This analysis showed a significant distance effect for binocular viewing $[F(4,36)=48.17, p<.01]$, but the effect for monocular viewing was nonsignificant $[F(4,36)=.58, p>.05]$. Thus, it was clear that changes in viewing distance led to changes in binocular accommodation, but not in monocular accommodation.

Relationships among DF and monocular and binocular accommodation are expressed on the right side of Table 2. Monocular accommodation was significantly correlated with DF at all viewing distances, as was binocular accommodation at all distances, except $25 \mathrm{~cm}$. Again, it would appear that the influence of DF was stronger on monocular than on binocular accommodation. A 2 by 5 (Ocularity by Target Distance) analysis of variance, with the dependent variable being the absolute difference (i.e., signs ignored) between such subject's accommodation under a given condition and that subject's DF baseline, showed a significant effect due to ocularity $[F(1,9)=$ $9.66, p<.02]$. In other words, as was the case in Experiment 1, binocular viewing led to accommodation values significantly more divergent from DF than did monocular viewing. The overall effect due to target distance was not significant, but the interaction was $[F(4,36)=2.93, p<.05]$.

\section{Discussion}

From the results of Experiment 2, it can be seen that it is possible to construct a workable, while at the same time fairly simple and inexpensive, openloop system for stimulating changes in ocular vergence without providing a direct sensory stimulus to accommodation. It can also be seen from examining the results of both Experiment 1 and Experiment 2 that not just any small stimulus will serve. In order to be free from accommodation-producing cues, the target stimulus apparently has to be extremely impoverished at viewing distances closer than $50 \mathrm{~cm}$. 
Leibowitz and Owens (1975) were not incorrect in their conclusion that at distances of 50 and $400 \mathrm{~cm}$ even a relatively large target light (in their case, $1.2 \mathrm{~mm}$ ) is insufficient to induce accurate monocular accommodation. This conclusion was confirmed in Experiment 1. The problem develops at closer viewing distances, when the stimulus must be much more carefully controlled to prevent it from serving as an accommodative stimulus. Fincham and Walton's (1957) assumption that a stimulus of $.5 \mathrm{~mm}$ was not sufficient to induce accommodation is thus somewhat suspect. This may account for the fact that two of Fincham and Walton's three subjects showed a very high degree of correspondence between binocular accommodation and convergence distance at very close distances, closer correspondence than was found in either of the two experiments described in the present report. Fincham and Walton's subjects may have been accommodating directly to the sensory information provided by the target. Since Fincham and Walton reported such data for only three subjects, however, it is difficult to draw convincing conclusions.

A question that is not answered by the present experiments is: Why was the target stimulus used in Experiment 2 not an effective cue for accommodation? Perhaps the visual system is unable to detect the changes in spatial contrast that result from defocus of a point source, similar to the situation encountered with interference patterns or small pupils (Owens, 1980). Contributing to this difficulty might be the additional blur provided by the ground placed on the observer's side of the pinhole. Or perhaps it is the extreme dimness of the target that makes it ineffective for inducing accommodation, rather than the small size per se. Other research will have to answer this question.

Both experiments provide additional support for the position that ocular vergence can drive accommodation. Of course, since these experiments were conducted under highly artificial viewing conditions (i.e., total darkness), it would be going considerably beyond the data to maintain that ocular vergence does drive accommodation under normal viewing circumstances (as Fincham \& Walton, 1957, have suggested). Nevertheless, the data from these experiments do support the possibility that such could be the case. These data also serve to point out that in studies in which the voluntary nature of accommodation is being examined, ocular vergence must be accounted for. As pointed out in the introduction to this report, it is possible that what is being voluntarily controlled is ocular vergence and accommodation is just following along.

The results of these two experiments again emphasize the importance of DF in reduced illumination viewing conditions. Both monocular and binocular accommodation seem to be related to DF, as shown in
Table 1, and this relationship seems to get stronger as the target becomes weaker (i.e., from Experiment 1 to Experiment 2). This, of course, confirms previous findings (e.g., Owens \& Leibowitz, 1975, 1976) and emphasizes the importance of binocular vision in maintaining accurate accommodation under reduced illumination viewing conditions. As mentioned in the introduction to the present report, DF appears to be a constant that exerts an influence on many different kinds of visual functioning.

Another finding of interest in these two experiments is that observers can apparently converge to an impoverished target under reduced illumination conditions. Several studies (Fincham, 1962; Ivanoff, 1955; Owens \& Leibowitz, 1976) have shown that in the absence of a convergence stimulus and/or in greatly reduced illumination, ocular vergence assumes an intermediate position analogous to DF. The results of the present experiments, however, seem to indicate that it does not require much of a stimulus to produce ocular vergence changes effectively. Such a conclusion is based on inference from the accommodation data, of course, as ocular vergence was not directly measured. It must also be remembered that subjects in the present experiments were carefully screened for phorias, something that has not always been done in previous experiments, and the results may not, as a result, be representative of the population as a whole. Nevertheless, it seems likely that ocular vergence can be quite responsive to even very impoverished cues.

Finally, Experiment 2 provides a result of some importance to researchers investigating the various parameters of DF. One concern in doing lengthy experiments in DF is that fatigue effects, induced by the experiment itself, might affect DF. In Experiment 2 , it was clear that no such effects occurred, as the second baseline measure was not significantly different from the first. Since the time that elapsed between the first and last measure was typically between 50 and $60 \mathrm{~min}$, DF appears to be fairly robust with regard to fatigue effects.

\section{REFERENCES}

Charman, W. N. On the position of the plane of stationarity in laser refraction. American Journal of Optometry and Physiological Optics, 1974, 51, 832-838.

Fincham, E. F. Accommodation and convergence in the absence of retinal images. Vision Research, 1962, 1, 425-440.

Fincham, E. F., \& Walton, J. The reciprocal actions of accommodation and convergence. Journal of Physiology, 1957, 137, 488-508.

GOGEL, W. C. Scaler perceptions with binocular cues of distance. American Journal of Psychology, 1972, 85, 477-497.

Hennessy, R. T. Instrument myopia. Journal of the Optical Society of America, 1975, 65, 1114-1120.

Hennessy, R. T., Iida, T., Shina, K., \& Leibowitz, H. W. The effects of pupil size on accommodation. Vision Research, $1976,16,587-589$.

Hennessy, R. T., \& Leibowitz, H. W. Subjective measurement 
of accommodation with laser light. Journal of the Optical Society of America, 1970, 60, 1700-1701.

Hennessy, R. T., \& Leibowitz, H. W. Laser optometer incorporating the Badal principle. Behavior Research Methods \& Instrumentation, 1972, 4, 237-239.

IvaNOFF, $A$. Night binocular convergence and night myopia. Journal of the Optical Society of America, 1955, 45, 769-770.

LEIBowitz, H. W., \& Hennessy, R. T. The laser optometer and some implications for behavioral research. American Psychologist, 1975, 30, 349-352.

Leibowitz, H. W., Hennessy, R. T., \& Owens, D. A. The intermediate resting position of accommodation and some implications for space perception. Psychologia, 1975, 18, 162-170.

Leibowitz, H. W., \& OWens, D. A. Anomalous myopias and the intermediate dark focus of accommodation. Science, 1975, 189, 646-648.

Leibowitz, H: W., \& Owens, D. A. New evidence for the intermediate position of relaxed accommodation. Documenta Ophthalmologica, 1978, 46, 133-147.

Malmstrom, F. V., \& Randle, R. J. Effects of visual imagery on the accommodation response. Perception \& Psychophysics, $1976,19,450-453$.

Miller, R. J. Temporal stability of the dark focus of accommodation. American Journal of Optometry and Physiological Optics, 1978, 55, 447-450. (a)

Miller, R. J. Mood changes and the dark focus of accommodation. Perception \& Psychophysics, 1978, 24, 437-443. (b)

Morgan, M. W. Accommodation and vergence. American Journal of Optometry and Archives of American Academy of Optometry, 1968, 45, 417-454.

Ogle, K. N. Optics: An introduction for ophthalmologists (2nd ed.). Springfield, Ill: Thomas, 1968.

OWENS, D. A. A comparison of accommodative responsiveness and contrast sensitivity for sinusoidal gratings. Vision Research, $1980,20,159-167$.

Owens, D. A., \& Leibowitz, H. W. The fixation point as a stimulus for accommodation. Vision Research, 1975, 15, 1161-1163.

OWens, D. A., \& Leibowitz, H. W. Oculomotor adjustments in darkness and the specific distance tendency. Perception \& Psychophysics, 1976, 20, 2-9.

Provine, R. R., \& Enoch, J. M. On voluntary ocular accommodation. Perception \& Psychophysics, 1975, 17, 209-212.

WESTHEIMER, G. Accommodation measurements in empty visual fields. Journal of the Optical Society of America, 1957, 47, 714-718.

Whiteside, T. C. D. Accommodation of the human eye in a bright and empty visual field. Journal of Physiology (London), $1952,118,65 \mathrm{P}$

\section{NOTE}

1. While there was some slight tendency for subjects to accommodate more to the near target than to the far target, the correlations between subjects' individual DF values and their actual accommodation to the target had coefficients of .92 for the $50-\mathrm{cm}$ target and .90 for the $400-\mathrm{cm}$ target. The original article (Owens \& Leibowitz, 1975) did not report any significant difference between near-target and far-target accommodation, but a personal communication from Dr. Owens to the present author (November 1979) indicated that this difference was statistically significant $[t(10)=7.25, p<.01]$.

(Received for publication December 7, 1979; revision accepted May 7, 1980.) 